\title{
Molecular Events During Resting Cyst Formation in Unicellular Eukaryote Colpoda
}

\author{
Tatsuomi Matsuoka* \\ Department of Biological Science, Faculty of Science \& Technology, Kochi University, Japan \\ *Corresponding author: T Matsuoka, Department of Biological Science, Faculty of Science \& Technology, Kochi University
} 780-8520, Japan

\section{ARTICLE INFO}

Received: 蔧 November 20, 2019

Published: 幽 December 04, 2019

Citation: Tatsuomi Matsuoka. Molecular Events During Resting Cyst Formation in Unicellular Eukaryote Colpoda. Biomed J Sci \& Tech Res 23(3)-2019. BJSTR. MS.ID.003916.

Keywords: Colpoda; Resting Cysts; Encystment; $\mathrm{Ca}^{2+} /$ Calmodulin; cAMP; Phosphorylation

\section{ABSTRACT}

An understanding of intracellular signaling pathways leading to resting cyst formation (encystment) is essential for the development of clinical drugs to prevent the life cycle of pathogenic unicellular eukaryotes. Recent studies imply that there are common signaling pathways among pathogenic and nonpathogenic unicellular eukaryotes. This paper describes molecular events, including signaling pathways, in the encystment of the nonpathogenic unicellular eukaryote Colpoda, based on results obtained mainly in our laboratory in the past 20 years.

Abbreviations: $\mathrm{Ca}^{2+} / \mathrm{CaM}: \mathrm{Ca}^{2+} /$ calmodulin; UV: Ultraviolet rays; PKA: Protein kinase A; Phos-tag/ECL: Phosphate-binding tag/enhanced chemiluminescence; LC-MS/MS: Liquid chromatography-tandem mass spectrometry; 2-D PAGE: Two-dimensional polyacrylamide gel electrophoresis; SDS-PAGE: Sodium dodecyl sulfate-polyacrylamide gel electrophoresis: EF-1 $\alpha$ : Elongation factor $1 \alpha$; AMPK: AMP-activated protein kinase; eEF2K : Eukaryotic Elongation Factor-2 Kinase
\end{abstract}

\section{Introduction}

One strategy of some pathogenic unicellular eukaryotes is to form resting cysts that are resistant to the environmental stresses of the host, such as stomach acid or immune system attack. Therefore, an understanding of intracellular signaling pathways leading to resting cyst formation (encystment) is essential for the development of clinical drugs to prevent cyst formation. It has been reported that the induction of the encystment of pathogenic Entamoeba histolytica is $\mathrm{Ca}^{2+} /$ calmodulin $\left(\mathrm{Ca}^{2+} / \mathrm{CaM}\right)$-dependent [1] and cAMP-dependent in the pathogenic unicellular eukaryote Giardia [2]. $\mathrm{Ca}^{2+} / \mathrm{CaM}$ and cAMP are also essential components in intracellular signaling pathways of the nonpathogenic free-living soil ciliate Colpoda [3]. These facts imply that common signaling pathways leading to encystment occur in some pathogenic and nonpathogenic unicellular eukaryotes. Recently, early molecular events during the encystment of Colpoda, including signaling pathways, were elucidated extensively. In this paper, we describe the encystment process of Colpoda based mainly on our research results. In hazardous environments, Colpoda promptly transforms into resting cysts that are resistant to desiccation, freezing, high temperature, acid, and UV light [4-7]. In the case of Colpoda cucullus Nag-1 [8], encystment can be induced by suspending vegetative Colpoda cells at a high cell density in the presence of $\mathrm{Ca}^{2+}[3]$.

\section{Morphogenetic Events During Encystment of C. cucullus Nag-1}

The morphogenetic changes in encystment-induced Colpoda cells are described as follows:

2 3 h after Encystment Induction: The cells stop swimming and begin to aggregate by an excretion of sticky mucus. The cells then round up, and then small sticky globules showing a fibrous or crystal-like fine structure [9] called lipidosomes [10] are extruded to be trapped by the mucus layer, followed by the formation of an ectocyst layer (a rigid single-cyst wall layer) [9]. In this stage, the cellular structures characterizing vegetative cells such as cilia begin to disintegrate and mitochondrial activity is arrested [11].

3 5 $\mathbf{~ h}$ after Encystment Induction: At 3 $5 \mathrm{~h}$ after encystment induction, the first-synthesized endocyst layer is formed by the excretion and gelation of an endocyst-precursor substance between 
an ectocyst layer and the plasma membrane [9]. In order to digest vegetative structures, several autophagosomes are formed to digest the vegetative structures.

5 h 1 week after Encystment Induction: The formation of endocyst layers is repeated (presumably twice per day), and several layers of endocyst are formed in several days. Auto phagocytosis is nearly completed within $24 \mathrm{~h}$. In the 1 -week-old mature cyst, the cytoplasm is filled with an amorphous electron-lucent material and many electron-lucent ellipsoidal granules (presumably reserve grains) accumulate [9].

\section{Signaling Pathways Leading to Encystment and Early Molecular Events During Resting Cyst Formation}

Using a pharmacological method, we have proposed that intracellular signaling pathways leading to the encystment of $C$. cucullus Nag- 1 are activated by $\mathrm{Ca}^{2+}$-calmodulin, followed by an increase in intracellular cAMP concentrations [3]. Thereafter, an encystment-dependent elevation of the intracellular $\mathrm{Ca}^{2+}$ concentration was demonstrated by using Fura 2 ratiometry [12]. Intracellular $\mathrm{Ca}^{2+}$ may activate calmodulin, which is thought to activate adenylate cyclase to elevate cAMP, followed by activation of protein kinase A (PKA) [3]. Actually, cAMP enzyme immunoassay (EIA) showed up to ten-fold elevation of cAMP levels in encystment-induced Colpoda cells $[13,14]$. cAMP-dependently phosphorylated proteins were detected by means of biotinylated Phos-tag/enhanced chemiluminescence (ECL) assay [15] and then identified by a liquid chromatography-tandem mass spectrometry (LC-MS/MS) analysis $[16,17]$. The proteins whose phosphorylation levels are up-regulated at $1 \mathrm{~h}$ after encystment is induced by $\mathrm{Ca}^{2+} /$ overpopulation of Colpoda vegetative cells or by the addition of membrane-permeation-type cAMP acetoxymethyl ester (cAMPAM) are listed as follows (most phosphorylated proteins are common in $\mathrm{Ca}^{2+}$ /overpopulation induction and cAMP induction):

a. Ribosomal P0 protein (localized in macronucleus)

Presumed function: Regulation of gene expression and metabolism

b. Ribosomal S5 protein

Presumed function: Arrest of cell cycle

c. Rieske iron-sulfur protein (RISP)

Presumed function: Arrest of mitochondrial activity

d. Histone H4 (hyperacetylated form)

Presumed function: Chromatin condensation of the macronucleus

e. Actin

Presumed function: Resorption of cilia

During Colpoda encystment, the expression of encystmentspecific proteins is expected to occur, and most proteins expressed in vegetative cells may be silenced. Actually, mRNA levels are extremely reduced within $5 \mathrm{~h}$ after encystment induction [11]. We analyzed the alteration of water-insoluble protein expression levels by sodium dodecyl sulfate-polyacrylamide gel electrophoresis (SDSPAGE) or two-dimensional polyacrylamide gel electrophoresis (2-D PAGE) and identified these proteins by LC-MS/MS analysis $[11,18]$. The proteins whose expression levels are modified within $12 \mathrm{~h}$ after encystment induction are listed as follows:

a. Elongation factor $1 \alpha$ (EF-1 $\alpha)$ [up-regulated 3h after encystment induction]

Presumed function: Acceleration of protein synthesis

b. ATP synthase $\quad \beta$ chain [downregulated $4 \mathrm{~h}$ after encystment induction]

Presumed function: Disappearance of the mitochondrial membrane potential

c. Heat shock protein 60 (HSP 60) [temporarily up regulated $5 \mathrm{~h}$ after encystment induction]

Presumed function: Molecular chaperon

d. Actin-related $49 \mathrm{kD}$ protein [up-regulated $1.5 \mathrm{~h}$ after encystment induction]

Presumed function: Chromatin remodeling

The 2-D PAGE of water-insoluble components (containing ciliary microtubules) of $C$. cucullus Nag-1 showed that the amount of tubulin is drastically reduced within several hours after the onset of encystment induction [11]. In this case, tubulin gene expression may not be downregulated but instead probably resulted from the disassembly of microtubules of ciliary axoneme. Knowledge of the signaling pathways for encystment, including those proposed by our research group, has been extensively advanced by the Colpoda aspera transcriptome analysis performed by Jiang et al. [19]. In the signaling pathways activated by $\mathrm{Ca}^{2+}$-calmodulin, the expression of genes for AMP-activated protein kinase (AMPK), eukaryotic elongation factor-2 kinase (eEF2K), AKT (protein kinase B) and several genes for autophagy is up-regulated.

\section{Conclusion}

Although Colpoda is a nonpathogenic unicellular eukaryote, an understanding of molecular events in the early stages of Colpoda encystment is expected to help elucidate the signaling pathways leading to the encystment of pathogenic unicellular eukaryotes.

\section{Acknowledgment}

This research was financially supported by a Sasagawa Scientific Research Grant (\#24-407) from Japan Science Society, by a Research Fellowship of the Japan Society for the Promotion of Science for Young Scientists (\#13J08784), and by Grant-in-Aid for Scientific Research (B) (\#19H03447). 


\section{References}

1. Makioka A, Kumagai M, Ohtomo H Kobayashi S, Takeuchi T (2001) Effect of calcium antagonists, calcium channel blockers and calmodulin inhibitors on the growth and encystations of Entamoeba histolytica and E. invadens. Parasitol Res 87(10): 833-837.

2. Gibson C, Schanen B, Chakrabarti D, Chakrabarti R (2006) Functional characterisation of the regulatory subunit of cyclic AMP-dependent protein kinase A homologue of Giardia lamblia: Differential expression of the regulatory and catalytic subunits during encystation. Int J Parasitol 36(7): 791-799.

3. Matsuoka T, Kondoh A, Sabashi K, Nagano N, Akematsu T, et al. (2009) Role of $\mathrm{Ca}^{2+}$ and cAMP in a cell signaling pathway for resting cyst formation of ciliated protozoan Colpoda cucullus. Protistology 6: 103110.

4. Taylor CV, Strickland AGR (1936) Effects of high vacua and extreme temperatures on the cysts of Colpoda cucullus. Physiol Zool 9(1): 15-26.

5. Maeda H, Akematsu T, Fukui R, Matsuoka T (2005) Studies on the resting cyst of ciliated protozoan Colpoda cucullus: Resistance to temperature and additional inducing factors for en-or excystment. J Protozool Res 15(1-2): 7-13.

6. Sogame Y, Kida A, Matsuoka T (2011) Possible involvement of endocyst in tolerance of the resting cyst of Colpoda cucullus against $\mathrm{HCl}$. African J Microbiol Res 5(25): 4316-4320.

7. Matsuoka K, Funadani R, Matsuoka T (2017) Tolerance of Colpoda cucullus resting cysts to ultraviolet irradiation. J Protozool Res 27(1-2): $1-7$

8. Funadani R, Sogame Y, Kojima K, Takeshita T, Yamamoto K, et al. (2016) Morphogenetic and molecular analyses of cyst wall components in the ciliated protozoan Colpoda cucullus Nag-1. FEMS Microbiol Lett 363(18): fnw203.

9. Funatani R, Kida A, Watoh T, Matsuoka T (2010) Morphological events during resting cyst formation (encystment) in the ciliated protozoan Colpoda cucullus. Protistology 6: 204-217.

10. Foissner W, Stoeck T, Agatha S, Dunthorn M (2011) Intraclass evolution and classification of the Colpodea (Ciliophora). J Eukaryot Microbiol 58(5): 397-415.

ISSN: 2574-1241

DOI: 10.26717/BJSTR.2019.23.003916

Tatsuomi Matsuoka. Biomed J Sci \& Tech Res

(C) This work is licensed under Creative

Submission Link: https://biomedres.us/submit-manuscript.php
11. Sogame Y, Kojima K, Takeshita T, Kinoshita E, Matsuoka T (2014) Identification of differentially expressed water-insoluble proteins in the encystment process of Colpoda cucullus by two-dimensional electrophoresis and LC-MS/MS analysis. J Eukariot Microbiol 61(1): 5160.

12. Sogame Y, Matsuoka T (2013) Evaluation of intracellular $\mathrm{Ca}^{2+}$ concentration by fura 2 ratiometry in encystment-induced Colpoda cucullus. Acta Protozool 52(1): 51-54.

13. Asami H, Ohtani Y, Iino R, Sogame Y, Matsuoka T (2010) Behavior and $\mathrm{Ca}^{2+}$-induced cell signaling for encystment of Colpoda cucullus. J Protozool Res 20(2): 1-6.

14. Sogame Y, Kinoshita E, Matsuoka T (2011) $\mathrm{Ca}^{2+}$-dependent in vivo protein phosphorylation and encystment induction in the ciliated protozoan Colpoda cucullus. Eur J Protistol 47(3): 208-213.

15. Kinoshita E, Kinoshita Kikuta E, Takiyama K, Koike T (2006) Phosphatebinding tag: A new tool to visualized phosphorylated proteins. Mol Cell Proteomics 5(4): 749-757.

16. Sogame Y, Kojima K, Takeshita T, Fujiwara S, Miyata S, et al. (2012) Protein phosphorylation in encystment-induced Colpoda cucullus: localization and identification of phosphoproteins. FEMS Microbiol Lett 331(2): 128-135.

17. Sogame Y, Kojima K, Takeshita T, Kinoshita E, Matsuoka T (2014) Identification of cAMP-dependent phosphorylated proteins involved in the formation of environment-resistant resting cysts by the terrestrial ciliate Colpoda cucullus. Inv Surv J 11: 213-218.

18. Sogame Y, Kojima K, Takeshita T, Kinoshita E, Matsuoka T (2012) EF-1 $\alpha$ and mitochondrial ATP synthase $\beta$ chain: alteration of their expression in encystment-induced Colpoda cucullus. J Eukaryot Microbiol 59(4): 401-406.

19. Jiang C, Wei W, Yan G, Shi T, Miao W (2019) Transcriptome analysis reveals the molecular mechanism of resting cyst formation in Colpoda aspera. J Eukaryot Microbiol 66(2): 212-220.

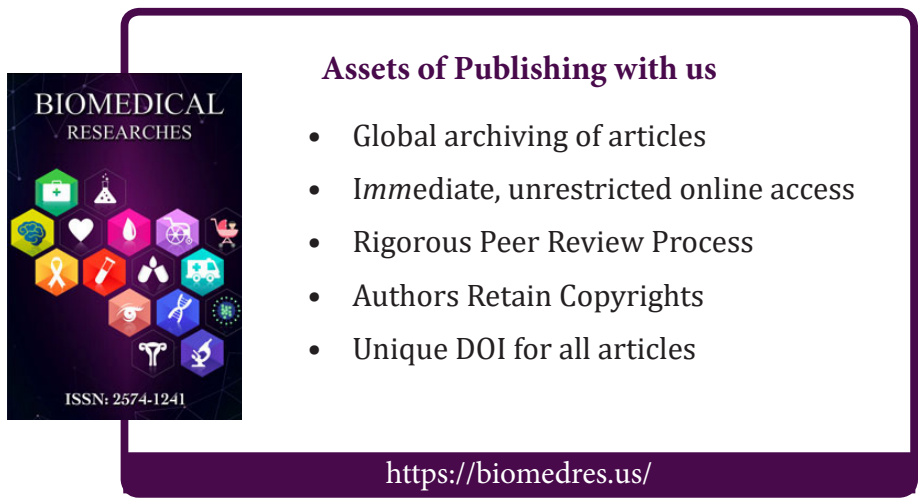

